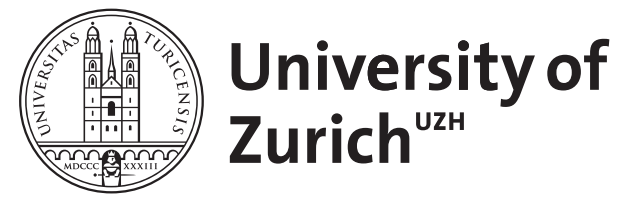

Zurich Open Repository and Archive

University of Zurich

University Library

Strickhofstrasse 39

CH-8057 Zurich

www.zora.uzh.ch

Year: 2018

\title{
Denkbewegungen. Philosophische Reflexion einer soziologischen Kritik
}

Weiner, Sebastian

DOI: https://doi.org/10.14361/9783839443620-012

Posted at the Zurich Open Repository and Archive, University of Zurich

ZORA URL: https://doi.org/10.5167/uzh-156810

Book Section

Published Version

Originally published at:

Weiner, Sebastian (2018). Denkbewegungen. Philosophische Reflexion einer soziologischen Kritik. In:

Henkel, Anna; Behrendt, Gianna. 10 Minuten Soziologie : Fakten. Bielefeld: transcript, 147-158.

DOI: https://doi.org/10.14361/9783839443620-012 


\section{Denkbewegungen \\ Philosophische Reflexion einer soziologischen Kritik}

\section{Sebastian Weiner}

\section{Philosophie und Soziologie}

Im Nachfolgenden geht es darum, eine berechtigte soziologische Kritik an der philosophischen Methode wiederum philosophisch zu reflektieren. Dieses Vorgehen ist schon deshalb ungewöhnlich, weil die Philosophie bemerkenswert wenig Interesse an der Soziologie zeigt, während sie offen ist etwa für Diskussionen aus der Linguistik, Neurologie und Biologie. Dieser Tatbestand ist dann als Missstand zu werten, wenn der Philosophie dadurch etwas entgeht. Der nachfolgende Text wird erstens exemplarisch zeigen, dass der Tatbestand tatsächlich vorliegt, zweitens, weshalb dies einen Missstand für die Philosophie darstellt und drittens, weshalb die Soziologie mit ihrer Empfehlung, wie er zu beheben ist, an der Sache vorbeizielt.

Wie ist das methodisch einzuordnen? Die Soziologie scheint sich durch etwas auszuzeichnen, was Luhmann für die modernen Wissenschaften geltend macht, nämlich das Beobachten des Beobachtens. Sie versucht hierzu, den Gegenstand der Beobachtung möglichst nah heranzuholen. Die Philosophie hingegen lebt seit jeher von der Abstraktion, die mit Blick auf das Objekt zwar ein Herausziehen des Wesentlichen in allem Unwesentlichen sein soll, zugleich aber vom Subjekt her gesehen ein Zurücktreten meint, um das Ganze in den Blick zu bekommen.

Der vorliegende Beitrag wird sich daher mit einer soziologischen Theorie befassen, die der Philosophie bei ihrem Treiben über die Schulter schaut und hierzu möglichst nah herangegan- 
gen ist an das zu beobachtende Beobachten. Die anschließende philosophische Kritik an dieser Theorie wird versuchen, das in der soziologischen Theorie Empfohlene möglichst allgemein $\mathrm{zu}$ reflektieren, um dessen Richtigkeit zu prüfen. Den LeserInnen wird also zugemutet, erst möglichst nah an das Treiben der Philosophie heranzutreten, um es dann wieder mit Abstand zu betrachten.

\section{Der Tatbestand: Ignoranz der Philosophie gegenüber soziologischer Kritik}

Der Tatbestand, dass sich die Philosophie nicht mit soziologischen Diskussionen befasst, lässt sich exemplarisch für einen soziologischen Autor zeigen, dessen Kritik an der Philosophie für sie von Wichtigkeit wäre. Pierre Bourdieus im Jahre 2010 auf Deutsch erschienenes Buch »Meditationen - Zur Kritik der scholastischen Vernunft (im frz. Original »Méditations pascaliennes«, 1997) ergründet die Bedingungen der Möglichkeit des philosophischen Denkens. Bourdieus von Kant entliehener Ausdruck der >Bedingung der Möglichkeit` deutet bereits darauf hin, dass sich die Philosophie ihrer sozialen Bedingungen nicht bewusst ist. Wenn Bourdieu Recht hat, befindet sich die Philosophie, um sich verbal wieder an Kant anzulehnen, in einem metaphysischen Schlummer, aus dem sie dringend erweckt werden sollte.

In der Soziologie ist Bourdieus Bedeutung groß. Laut einer Umfrage der International Sociological Association (www.isasociology.org) zählt Bourdieus Buch »Distinction: A Social Critique of the Judgment of Taste« (im frz. Original: »La Distinction. Critique sociale du jugement«) zu den für die Soziologie einflussreichsten Büchern des 20. Jahrhunderts (Rang 6). Doch weder diese Popularität noch seine explizite Kritik an der Philosophie hat einen Einfluss auf seine Wirkung in dieser Disziplin. Nur der Vollständigkeit halber sei angemerkt, dass das, was hier 
für Bourdieu aufgezeigt wird, sich ebenso signifikant in Bezug auf Luhmann ergibt. Auch von ihm könnte die Philosophie viel lernen, vermeidet es aber beharrlich, sich näher mit ihm zu befassen.

Um den subjektiven Eindruck empirisch zu untermauern, dass Bourdieus gesamtes Werk und Denken in die Philosophie so gut wie keinen Eingang gefunden hat, lassen sich die Suchergebnisse der für philosophische Zeitschriften wichtigsten Datenbank nutzen: www.jstor.org. Hier sind mehr als Zweidrittel der international renommierten philosophischen Fachzeitschriften vertreten.

Durchsucht man sämtliche Artikel aus 131 philosophischen Fachzeitschriften nach Beiträgen, in deren Titel »Bourdieu« vorkommt, erhält man 17 Treffer, davon 16 in der Zeitschrift "Sociological Theory« und einen in »Political Theory« (ein Aufsatz zu Hannah Arendt und Pierre Bourdieu). Geht man davon aus, dass beide Organe keine genuin philosophischen Zeitschriften sind, ergibt sich eine Trefferquote von Null. Kein Aufsatz in einer philosophischen Fachzeitschrift bezieht sich im Titel explizit auf Bourdieu.

Eine Volltextsuche nach »Bourdieu« inklusive Fußnoten in selbigen 131 Zeitschriftentiteln ergibt zwar 279 Treffer, doch ist die Ausbeute bei genauem Hinsehen gering. 186 Treffer entfallen auf die Zeitschrift »Sociological Theory«, 51 auf das »Journal of Business Ethics «, 18 auf das "Journal of Aesthetics and Art Criticism« und 16 auf»Political Theory«. Die verbleibenden acht Treffer finden sich in Zeitschriften, die durchaus als genuin philosophisch angesehen werden können, nämlich fünf auf »Philosophy East and West « und drei auf »Ethics".

Noch einmal ist $\mathrm{zu}$ betonen, dass es sich hier um eine Volltextsuche handelt. Bereits schon den bloßen Namen »Bourdieu « im Text anzuführen oder in Fußnote oder Bibliographie auf eine seiner Schriften zu verweisen, ergab einen Treffer. Letztlich ver- 
bleiben nur drei signifikante Treffer. Einmal wird Bourdieu mit mehreren Sätzen zitiert (es geht in dem Aufsatz um die Rolle der Zeit in der chinesischen Logik), einmal findet sich eine zweiseitige Diskussion zu Bourdieu im Zusammenhang mit dem kulturellen Kapital und einmal gibt es eine Bezugnahme auf ihn mit Blick auf politische Konflikte in Wohlfahrtgesellschaften (immerhin von der renommierten Philosophin Nancy Fraser). Die fünf übrigen Treffer sind lose Verweise auf Bourdieu oder Literaturangaben.

Um eine Vergleichsgröße zu erhalten, lässt sich bspw. »Foucault« in die Volltextsuche eingeben. Michel Foucault war dem eigenen Bekunden nach kein Philosoph, hat aber dennoch eine reiche philosophische Rezeption erfahren. Um Verzerrungen zu vermeiden, etwa durch Zeitschriften, die eine stark psychologische Ausrichtung haben (Foucaults Denken entstammt der Psychologie), wurden nur diejenigen Titel ausgewählt, die im SCImago Journal Rank unter den Top 20 gelistet und unter jstor. org abrufbar sind, unter Ausschluss der Titel »Political Psychology, European Child and Adolescent Psychiatry« und »Business Ethics Quarterly«. Damit verblieben für die Volltextsuche »Foucault« 15 Titel, die größtenteils eine sehr analytische Ausrichtung haben und daher Foucaults Denken eher fernstehen. Dennoch ergibt bereits diese eingeschränkte Suche 51 Treffer, im Vergleich zu den acht für Bourdieu, die sich in 127 Zeitschriftentiteln finden.

An dieser Stelle lässt sich einwenden, dass sich durch das Ergebnis in Bezug auf Foucault etwas Grundsätzliches zeigt, dass nämlich eine Datenbank wie jstor.org nicht geeignet ist, um die Rezeption von kritischen Denkern wie Foucault oder Bourdieu auch nur entfernt abzubilden. Das mag richtig sein, doch erstens gibt es keine alternative Datenbank, die weniger stark auf die angelsächsische Analytische Philosophie ausgerichtet ist, und zweitens geht es hier weniger um absolute Größen als um 
relative. Dem Ergebnis nach befasst sich die Philosophie zwar durchaus mit Foucault, aber so gut wie gar nicht mit Bourdieu, und dies entspricht dem subjektiven Eindruck. Damit von der Empirie zur normativen Betrachtung.

\section{Philosophische Blindheit und soziologische Kurzsichtigkeit}

Warum lohnt es sich für die Philosophie, sich mit der Soziologie zu befassen, und das meint, was entgeht ihr, wenn sie es nicht tut? Ihr entgeht eine kritische und damit reinigende Selbstreflexion, die sie selbst so nicht anstellen kann, weil ihr die Nähe zum Objekt fehlt, das in diesem Fall sie selbst ist. Bourdieu wirft der Philosophie vor, sie habe einen impliziten und illusorischen Anspruch auf Unbedingtheit (2010, S. 59):

»Die Philosophie hat keine Genese und kann keine haben; selbst wenn sie erst am Ende kommt, ist sie Beginn, und zwar radikaler Beginn, da sie mit einem Schlag als Totalität in Erscheinung tritt.«

Diese Beobachtung Bourdieus ist richtig. Zwar hat die Philosophie wenig Mühe, biologische, neuronale, linguistische oder vielleicht sogar soziale Bedingungen ihres Denkens anzuerkennen, jedoch nur, solange sie glauben darf, sich durch Abstraktion dieser entledigen zu können. Der Witz philosophischer Abstraktion besteht gerade darin, durch Herausarbeitung des Universalen das Kontingente und Historische loszuwerden und damit denkbare Bedingungen, die außerhalb des Denkens liegen. Daher liegt im Selbstverständnis der Philosophie ihre Genese zwar außerhalb des Denkens, aber ihre Geltung, d.h. ihr Begründungsanspruch, liegt dank der Abstraktion im reinen Denken. Bourdieu versucht nachzuweisen, dass auch der Be- 
gründungsanspruch sozial bedingt ist, und das gelingt ihm in Teilen bravourös. Aber er zieht daraus die falschen Schlüsse.

Die Philosophie braucht die vollständige Abstraktion, die Bourdieu als illusorisch ausgibt. Mit Blick auf die philosophische Methode, die darauf angelegt ist, von den lebensweltlichen Bedingungen der Möglichkeit des Denkens zu abstrahieren, kann es gar nicht verwundern, wenn Bourdieu Hegel und Heidegger $\mathrm{zu}$ Recht bescheinigt, für ihr eigenes Denken zeitlose und anfangslose Universalität zu beanspruchen, obwohl sie selbst die Historizität des Denkens aufgezeigt haben (2010, S. 60-62). Auch hat er Recht in Bezug auf die philosophische Hermeneutik: Sie unterlasse es zu fragen, ob ein bestimmtes Problem, das fester Bestandteil der philosophischen Tradition ist (z.B. das der Wahrheitsdefinition), mitunter erst durch eine bestimmte hermeneutische Tradition seine Geltung erhält. Pointierter noch als Bourdieu schreibt dazu Luhmann (2001, S. 266): »Dennoch hielt die Hermeneutik an der Idee fest, sie habe die Oberfläche eines Objekts (eines Textes) oder eines Subjekts (eines Bewusstseins) $\mathrm{zu}$ durchdringen, um in einer Tiefenschicht wahrheitsfündig $\mathrm{zu}$ werden.« Das ist alles richtig. Aber man muss die Philosophie eher dazu bringen, weiter in die Tiefe zu graben, statt ihr zu empfehlen, den Anspruch auf Tiefe aufzugeben. Oder anders gesagt: Sie hat noch immer nicht das Ganze in dem Blick bekommen, wie sie beansprucht.

Wenn sie in solche Tiefen nicht vordringen kann, dann kann es niemand, so ihr Selbstverständnis. Nietzsche schreibt in einer Notiz (Nietzsche, Montinari und Colli 1980, S. 53-54):

»Ich halte die Phänomenologie auch der inneren Welt fest: alles, was uns bewusst wird, ist durch und durch erst zurechtgemacht, vereinfacht, schematisiert, ausgelegt - der wirkliche Vorgang der inneren Wahrnehmung, die Causalvereinigung zwischen Gedanken, Gefühlen, Begehrungen, wie die zwischen Subjekt 
und Objekt, [ist] uns absolut verborgen - und vielleicht eine reine Einbildung."

Dass etwas anderes als die Philosophie diesen »wirklichen Vorgang « zum Vorschein bringt, hält Nietzsche für ausgeschlossen. Vielmehr ist es dann »absolut verborgen «. Doch der Schluss hieraus sollte nicht bedeuten, dass die Philosophie aufhören müsste, sich in solche Suchen zu versteigen. Sie würde aufhören, Philosophie zu sein.

Bourdieu wirft der Philosophie somit grundsätzlich vor, sich nicht mit den sozialen Bedingungen ihrer Möglichkeit auseinanderzusetzen, nämlich mit dem scholastischen Habitus, wie er es nennt. Stattdessen beharre sie auf ihrer Unbedingtheit und maße sich permanent an, »sich als (reine) Vernunft aus sich selbst zu begründen«, was eine »theoretische[n] Akrobatenleistung « darstelle, »die des Barons Münchhausen würdig wäre« (Bourdieu 2010, S. 57). Das, was Bourdieu als märchenhafte Akrobatenleistung darstellt, ist eine kritische Beschreibung dessen, was die Philosophie unter Abstraktion versteht.

Solche Kritik hat Tradition, und ebenso der falsche Schluss, den Bourdieu zieht. Im so genannten Streit um die Wissenssoziologie aus der ersten Hälfte des 20. Jahrhunderts, der sich ausgelöst durch Karl Mannheims Buch »Ideologie und Utopie« zwischen Philosophie und Soziologie entfachte, zeigt besonders die vermittelnde Position von Hannah Arendt deutlich, worum es ging.

Die Soziologie frage nach dem Ontischen, so Arendt, die Philosophie hingegen, allen voran Heidegger, nach dem Ontologischen. Die Soziologie beanspruche, "nach dem Seienden, das dieser [philosophischen] sSeinsauslegung zugrundeliegt, $\mathrm{zu}$ fragen, nach dem, was die Philosophie als das für sie Irrelevante behauptet « (1982, S. 516). Aus Sicht der Soziologie sei die philosophische Ontologie daher eine Ideologie. Um diese zu entlar- 
ven, sei es nötig, aufzuzeigen, »daß sie überhaupt qua Ontologie erst aus einer bestimmten Verdeckung, die das Seiende selbst geschaffen hat, entspringt « (1982, S. 517).

Das Wort »entspringen « ist hier mehrdeutig. Natürlich geht allem ontologischen Denken das Ontische voran. Ohne Menschsein und Lebenswelt auch keine philosophische Spekulation. Diesen Ursprung stellt keine Philosophie ernsthaft in Frage. Die Frage ist vielmehr, ob sich die Ontologie für ihre Geltung mittels Abstraktion aller ontischen Bedingungen und Verfärbungen entledigen kann, und hier sind Philosophie und Soziologie gegenteiliger Meinung. Die Philosophie glaubt, durch Abstraktion zum reinen Denken gelangen zu können, das zum Ursprung für alle weiteren philosophischen Überlegungen wird. Der Soziologe Bourdieu hingegen hat gezeigt, dass nicht nur die Genese der Philosophie, sondern auch ihre Geltung sozial bedingt ist, und ging damit vermutlich einen Schritt weiter als die Vertreter im Streit um die Wissenssoziologie.

Doch auch er glaubt, die einzige Therapie für die Philosophie laute Soziologie. So empfiehlt Bourdieu der Philosophie (2010, S. 65):

»Wer hier weiterkommen will, muß die von dem Höhlenmythos, dieser Berufsideologie der Berufsdenker, gerühmte Bewegung um - und zur Welt der Alltagsexistenz zurückkehren, gerüstet jedoch mit einer Intellektualität, die ihrer selbst und ihrer Grenzen hinreichend bewusst ist, um fähig zu sein, die Praxis zu reflektieren, ohne sie dabei zu eskamotieren.«

Bourdieu rät somit der Philosophie, von ihrem platonischen Weg hin zur Ontologie umzukehren und zur Lebenswelt zurückzukehren. Dabei hätte er sehen können: Wo dies geschehen ist, etwa bei Comte, Durkheim und Simmel, entstand daraus die So- 
ziologie. Die Philosophie kann hier nicht umkehren, ohne aufzuhören, Philosophie zu sein.

\section{Die Philosophie: Schon zu weit weg oder noch zu nah dran?}

Es lohnt sich, noch einmal genauer hinzusehen, was Bourdieu richtig gesehen hat und was nicht. Er hat Recht mit der Behauptung, die Philosophie begehe immer wieder den Fehler, Prinzipien anzunehmen, die nur vermeintlich dem reinen Denken entspringen. Eines von drei Beispielen, die Bourdieu hierzu anführt, ist Kants Idee des reinen Wohlgefallens als Prinzip für seine Ästhetik (Bourdieu 2010, S. 94-99). Wie Bourdieu an verschiedenen Stellen nachgewiesen hat, hängt die Fähigkeit zu reinem Wohlgefallen von einer bestimmten Sozialisation ab. Daher ist das reine Wohlgefallen gerade nichts rein Abstraktes, sondern etwas, das lebensweltliche Prägung hat. Kant hat etwas Lebensweltliches und historisch Bedingtes zum universalen Prinzip erhoben. Sein ästhetisches Prinzip und damit die Geltung seiner Aussagen sind historisch bedingt. Aus philosophischer Sicht war er noch zu nah dran an der Lebenswelt, aus soziologischer Sicht schon zu weit weg.

Dasselbe gilt vermutlich für Kants guten Willen und das Sittengesetz, auf denen er seine Ethik gründete. Dass diese womöglich einem protestantischen Habitus entspringen und damit lebensweltlich geprägt sind, konnte oder wollte er nicht sehen. Ähnlich ist zu fragen, ob Descartes cogito-Argument nicht nur denjenigen einleuchtet, die durch den Habitus der Berufsdenker gewohnt sind, der Realität des Denkens am allerwenigsten zu misstrauen.

Doch in diesen Fällen hat, anders als Bourdieu es glaubt, die Philosophie nicht zu viel von der Lebenswelt abstrahiert, sondern noch zu wenig. Sie ist noch nicht bis zum reinen Denken vorgedrungen. Aristoteles hatte Platon und seinen Anhängern vorge- 
halten, ihre durch Abstraktion gewonnene reine Form offenbare nur unzureichend die Natur der Dinge, denn diese bestünden notwendigerweise auch aus Materie (Physik II 1, S. 193b31-194a7). Der heutige Vorwurf seitens der Soziologie klingt ähnlich.

Platon war nie am Konkreten interessiert und ebenso wenig ist die heutige Philosophie an der Lebenswelt interessiert. Viel bedeutsamer wäre daher der Vorwurf gewesen, die Platoniker hätten noch zu wenig abstrahiert und die angebliche reine Form sei noch immer mit Konkretem kontaminiert. Bourdieu sagt ja genau dies, dass die Reinheit des reinen Wohlgefallens noch immer soziale Ursachen hat. Doch die Aufforderung, sich diesen zuzuwenden, sollte eine Aufforderung der Soziologie an die Soziologie sein und nicht eine an die Philosophie. Denn die Philosophie untersucht das Abstrakte (Begriffe, moralische Prinzipien, logische Prinzipien usw.) und nicht das Lebensweltliche.

Daher darf man auch nicht glauben, dass der Philosoph Wittgenstein seine eigene Zunft zum Blick auf die Lebenswelt hinwenden wollte, als er sie aufforderte (1984, S. 66): »Denk nicht, sondern schau!« Denn das Hinsehen, das Wittgenstein meint, ist im Grunde nur eine modifizierte Abstraktion. Am Ende dieser Abstraktion stehen nicht wie üblich Begriffe, sondern allgemeine Regeln (semantische, nicht soziale).

Die Empfehlungen, die Bourdieu mit Blick auf den philosophisch-scholastischen Habitus ausspricht, bleiben dementsprechend vage, da er zu ahnen scheint, dass die Hinwendung zur Lebenswelt zur Selbstaufgabe der Philosophie führen würde. Wenn er schreibt, das Erkennen des eigenen Habitus sei »ohne ein Wissen um sich selbst und seine eigene Praxis nicht zu haben«, das Voraussetzung sei, um "aufmerksamer und aufnahmebereiter für die tatsächlich praktizierte Praxis« zu werden (2010, S. 72), so stellt sich die Frage, ob die »tatsächlich praktizierte Praxis« nicht wieder die Abstraktion sein darf, die bislang noch nicht weit genug ging. 
Die Philosophie muss den Anspruch vertreten, dass sich Kontingenz und Historizität so reflektieren lassen, dass man sie erkennt und hinter sich lässt (selbst die Kontinentalphilosophie gesteht ja nicht zu, dass die Geltung ihrer Kritik und Dekonstruktion selbst wiederum nur sozial bedingt ist), weshalb die Philosophie einen Denker wie Foucault mit offenen Armen empfangen hat. Die Soziologie hingegen darf die Ansicht vertreten, dass dieser Anspruch bloße Illusion ist. Aber sie sollte nicht den Fehler begehen, die Philosophie zur Soziologie zu überreden, also zu einer Abwendung vom reinen Denken und Hinwendung zum Lebensweltlichen.

Abschließend drängt sich die Frage auf, ob die Philosophie nicht von selbst darauf kommen konnte, dass sie ihre noch lebensweltlich bedingten Prinzipien als reine ansieht. Vermutlich kann sie das nicht, denn um zu fragen, ob das durch Abstraktion Gewonnene tatsächlich von allem Lebensweltlichen gereinigt ist, muss man das Lebensweltliche kennen. Die Philosophie kann von der sozialen Bedingtheit der Geltung ihrer Prinzipien wenig wissen, weil sie sich generell nicht mit sozialen Bedingungen befasst. Sie ist ja gerade darum bemüht, von ihnen $\mathrm{Ab}$ stand zu nehmen. Das darf sie auch, solange sie bereit ist, sich von der Soziologie darüber aufklären zu lassen, dass ihr Denken doch nicht so rein ist, wie sie annahm. Dann bietet die Soziologie für die Philosophie einen echten Gewinn, nämlich den der Aufklärung.

Der Vollständigkeit halber sei angemerkt, dass die Idee, die Philosophie wolle die Dinge möglichst weit weg rücken, die Soziologie sie hingegen möglichst nah betrachten, von meinem soziologischen Lehrer Michael Makropoulos stammt. Diese Beobachtung der Beobachtung war für mein philosophisches Selbstverständnis recht hilfreich. 


\section{Zum Weiterlesen}

Bourdieu, Pierre (1982): Die feinen Unterschiede. Kritik der gesellschaftlichen Urteilskraft. Frankfurt a.M.: Suhrkamp.

Platon (2005): »Politeia, Buch VII«. In: Gunther Eigler (Hg.): Werke in acht Bänden. Bd. 4, Platon. Darmstadt:WBG.

Weiner, Sebastian (2016): Aristoteles' Bestimmung der Substanz als logos. Hamburg: Meiner.

\section{Literatur}

Arendt, Hannah (1982): »Philosophie und Soziologie«. In: Volker Meja/Nico Stehr (Hg.): Der Streit um die Wissenssoziologie, Bd.

2. Frankfurt a.M.: Suhrkamp, S. 515-531.

Aristotelis Physica, Hg. W.D. Ross, Oxford: Clarendon Press ([1936] 1956).

Bourdieu, Pierre (2010): Meditationen - Zur Kritik der scholastischen Vernunft. Frankfurt a.M.: Suhrkamp.

Luhmann, Niklas (2001): »Dekonstruktion als Beobachtung zweiter Ordnung«. In: Oliver Jahraus (Hg.): Aufsätze und Reden. Stuttgart: Reclam, S. 262-296.

Nietzsche, Friedrich/Montinari, Mazzino/Colli, Giorgio (1980): Kritische Studienausgabe, Bd. 13: Nachgelassene Fragmente 18871889. Berlin: de Gruyter.

Wittgenstein, Ludwig (1984): Philosophische Untersuchungen, Werkausgabe Bd. 1. Frankfurt a.M.: Suhrkamp. 